\title{
Interfacial crosslinked controlled thickness graphene oxide thin-films through dip-assisted
}

layer-by-layer assembly means

Vepika Kandjou, Miguel Hernaez, Beatriz Acevedo and Sonia Melendi-Espina* Engineering, Faculty of Science, University of East Anglia (UEA), Norwich, England (UK)

\section{GRAPHICAL ABSTRACT}

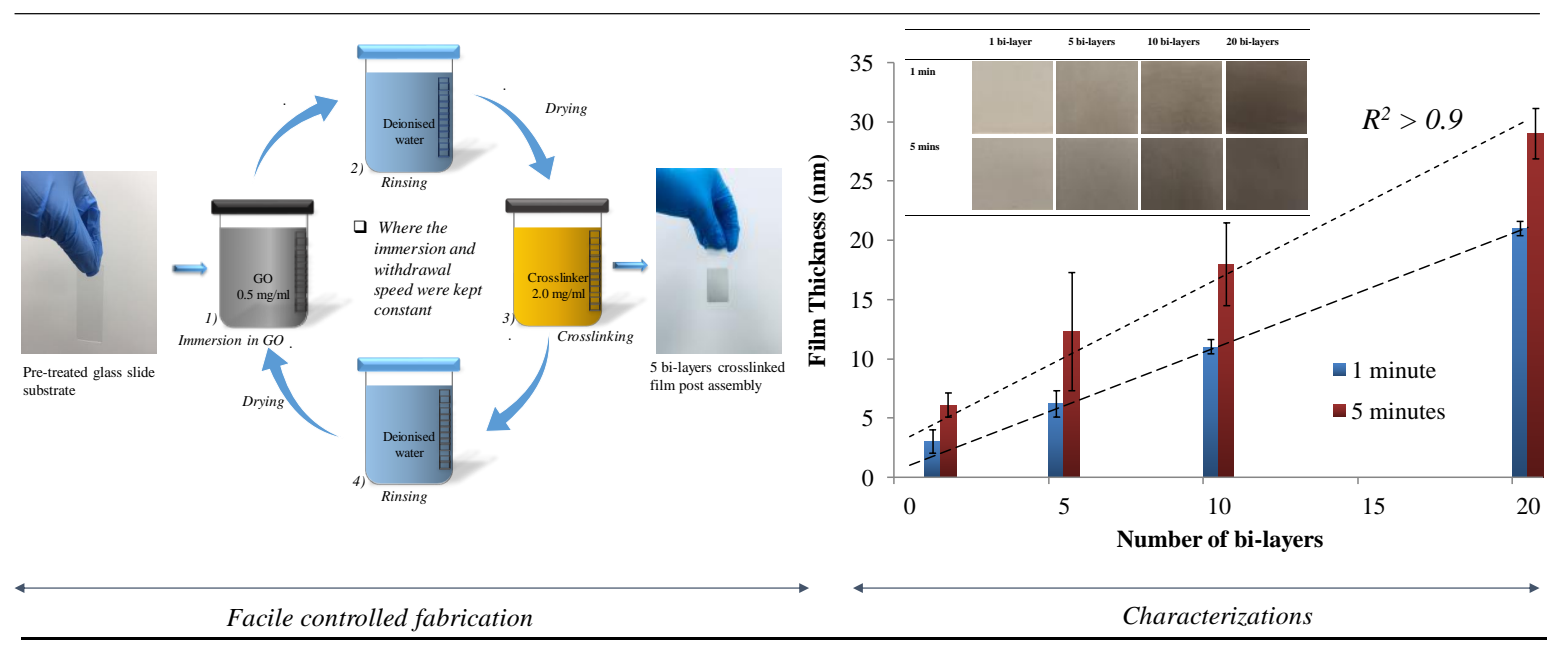

\section{ABSTRACT}

The augmentation of research in graphene based thin films has been of great interest to various current industrial stakeholders. This is mainly due to the wide scope of films applications, ranging from nanoelectronics to separation membranes. Therefore, establishing a relation between graphene based thin film key characteristics and the fabrication operating conditions is of high significance. This study entails the successful fabrication of controlled-thickness crosslinked graphene oxide (GO) thin films on inexpensive silicon-based glass slide substrates. The method of film fabrication used is the dip-assisted layer-by-layer assembly, which has an added advantage of step-control of thin film thickness, good film uniformity and continuity. The thickness was primarily tuned through the use different sized crosslinkers; a covalent based 
sub-nanometer sized p-phenylenediamine and an electrostatic based polyethyleneimine on an interchangeable assembly with GO. Pre film fabrication, Fourier Transform Infra-Red and XRay Photoelectron Spectroscopy characterizations were carried out to determine the nature of interactions between GO and the crosslinkers. Post film fabrication, scanning electron microscopy, water contact angle measurements and profilometry analysis were undertaken for film continuity, hydrophilicity and thickness measurements respectively. A strong linear trend between film thickness of the differently crosslinked films and the number of bi-layers was established.

\section{KEYWORDS}

Graphene oxide, layer-by-layer, crosslinking, thin film, thickness

\section{Introduction}

Thin films are of high importance in various applications, these varies from nanoelectronic optical instruments and sensors [1,2] to large scale operations like separation membranes and solar cells $[3,4]$. The effectiveness, durability and ease of operation of thin films in these various applications are dependent on their thickness, topographical roughness, continuity and homogeneity among other key characteristics [5]. Consequently, controlling the magnitude of these key characteristics is essential in thin film fabrication and operation [6].

From polymers to ceramics different materials have been employed in the fabrication of thin films for different purposes [7]. The recently discovered graphene is a great candidate material for usage as a thin film precursor because of its 2-dimentionality, mechanical strength and electrical properties $[7,8]$. However, despite its outstanding properties and promise in various applications, the use of graphene as a thin film precursor is limited by the material's lack of production scalability without compromising its structure, morphology and properties [9]. Low solubility of graphene in polar solvents is also a limitation to its usage as it cannot be easily dispersed in polar solvents to dispersions for the fabrication of thin films via solution requiring processes [10-13].

Owing to its ease of fabrication and large scale production scalability, graphene oxide (GO) has caught the attention of many researchers as a reasonable graphene alternative in the fabrication of thin films for various applications [14-20]. The most notable GO production 
method is the Modified Hummers method, which uses affordable graphite as a precursor raw material [21]. Although its mechanical and electrical properties are not as outstanding as those of graphene, in some applications like sensors and separation membranes the properties of GO are sufficient. This makes it a notable graphene substitute pertaining to these applications [2227]. Another key advantage of GO is its containment of oxygenated functional groups; the epoxy, hydroxyl and carboxylic groups, which make the material chemically active $[28,29]$. This means that it can be crosslinked with different molecules and thus improving the continuity of the GO based films via both vertical and horizontal crosslinking [30]. Crosslinking further introduces the ability to control the thickness, roughness and to enhance the stability of the fabricated films $[14,15,31]$.

Elsewhere different attempts to control film thickness and other key characteristics have been employed using various thin film fabrication techniques. For instance Shi-Hongfei et al. used spray coating to control film thickness via different spray volumes [32]. Green and Hersam on the other hand used solution phase production of graphene films and the thickness was controlled by density differentiation [33]. In this study the dip-assisted layer by layer assembly technique was entailed to ease the control and modification of key thin-film characteristics on a step by step basis.

The aforementioned methods are relatively costly in comparison to the dip-assisted layer-bylayer assembly [34]. The basis of this fabrication method is an interchangeable attachment of two or more materials on a layer-by-layer basis. The attachment can either be predominantly covalent based or electrostatic based depending on the nature and chemistry of the materials being assembled [14,15]. The layer-by-layer technique gives frameworks of controlled thickness through the use of different sized crosslinkers and operating conditions [35]. Specifically, dip-assisted layer-by-layer assembly offers cost effectiveness and ease of scale up to mass production as important advantages. The key operating conditions in dip assisted layerby-layer assembly are the immersion time, number of assembly cycles together with the immersion and withdrawal speeds [36].

Consequently, this study intended to demonstrate the fabrication of controlled thickness crosslinked GO films on affordable silicon-based glass slides to demonstrate the effectiveness of the dip assisted layer-by-layer assembly in controlled thin film thickness production. As such, two types of crosslinkers, a sub-nanometer sized covalent based p-phenylenediamine (PPD) and an electrostatic based polyethyleneimine (PEI) were used with GO. In specificity, this work aimed at evaluating the correlation between immersion times ( 1 and 5 minutes), 
number of bi-layers (1, 5, 10 and 20) significant thin film characteristics like; film thickness, continuity and hydrophilicity.

\section{Experimental Section}

\subsection{Materials}

GO powder (product code C889/GOB019/Pw2) was purchased from Graphenea Co, Spain. The crosslinkers; PPD powder (99\% product code: P6001) and $50 \%$ aqueous solution of PEI (product code: 03880) were both purchased from Sigma, Aldrich, UK. The films were fabricated on silicon-based substrates (CAT.NO.7101, 1.0-1.2mm thick) purchased from Fisher Scientific, UK. 1M of potassium hydroxide $(\mathrm{KOH})$ prepared from $\mathrm{KOH}$ powder (product code: $\mathrm{P} / 5640 / 53$ ) used for substrate pre-treatment was purchased from Fisher Scientific, UK as well.

\subsection{Study of interaction between GO and crosslinkers}

Prior to thin-film fabrication it was significant to determine the plausibility of GO - crosslinker interaction. The key to this was to firstly verify the presence of notable functional groups in GO and the crosslinkers and secondly to determine predominant interaction nature between GO and each of the crosslinkers.

Following preparation of the $0.5 \mathrm{mg} / \mathrm{ml}$ aqueous GO dispersion and $2.0 \mathrm{mg} / \mathrm{ml}$ of PPD and PEI aqueous solutions, each crosslinker was separately reacted with GO (1:1 ratio,) for 1 and 5 minutes. The reaction time was controlled by a $\mathrm{pH}$ switch after the designated reaction times through the addition of $99 \%$ sulphuric acid (Product code: 07208, Sigma Aldrich, UK), which resulted in the protonation of the reacting entities, hence rendering them unreactive $[37,38]$. The reacted samples were then centrifuged using a Bio-fuge Primo Heraeus centrifuge for 10 minutes at $6000 \mathrm{rpm}$ with thorough rinsing through a repetitive replacement of the supernatant with distilled water to remove the unreacted crosslinker and GO excess material.

The four centrifuge residue samples were then collected for Fourier Transform Infra-Red (ATR-FTIR PerkinElmer) and X-Ray Photoelectron Spectroscopy (Kratos Ultra-DLD XPS System (K-Alpha+) characterizations to verify GO-crosslinker interactions. For XPS characterizations wide-scan spectra in the binding energy range of approximately $0-1000 \mathrm{eV}$ were obtained to identify the elements present on the surfaces of the GO and the reacted entities. Additionally, high resolution spectra were obtained. Five peaks emerged when the C1s 
peak was curve fitted, corresponding to $\mathrm{C}$ graphitic (Binding energy, $\mathrm{BE},=284.3-284.4 \mathrm{eV}$ ), C-O epoxide/C-OH $(\mathrm{BE}=285.6-285.7 \mathrm{eV}), \mathrm{C}=\mathrm{O}$ carbonyl $(\mathrm{BE}=286.9-287.0)$, the band at 288.9 attributed to $\mathrm{COOH}$ carboxyl groups as well as the $\pi-\pi^{*}$ shake-up signal $(290.8 \mathrm{eV})$ typical for $\mathrm{sp}^{2}$ - hybridized carbon. It is worth it to mention that at these binding energies there is an overlap of oxygen and nitrogen functionalities, specifically between $\mathrm{C}$ (epoxy) and $\mathrm{C}=\mathrm{N}$ and among $\mathrm{C}=\mathrm{O}$ and $\mathrm{C}-\mathrm{N}[39-41]$.

\subsection{Thin film fabrication procedure}

To fabricate the GO - crosslinked films, the glass slide substrates were pre-treated, first by hydroxylation through immersion in $1 \mathrm{M} \mathrm{KOH}$ for 30 minutes to instigate a negative charge onto the slides though the formation of hydroxyls [42]. This was followed by thorough rinsing with deionized water and drying. The hydroxylated slides were subsequently immersed in a positively charged $2.0 \mathrm{mg} / \mathrm{ml}$ aqueous PEI for 5 minutes in order to instigate a positive charge on the glass slides [43]. The positively charged glass slides were then rinsed in deionized water and then dried before dip-assisted layer-by-layer assembly, which commenced with immersion in the negatively GO dispersion (Figure 1).

A rotary dip-coater device (Nadetech Innovations, Spain) (ND-R 11/2, S/N: 522016) was used to fabricate the films on an automated interchangeable dipping basis between GO and the crosslinkers (Figure 1). This process was done with rinsing of the glass slides in deionized water and drying prior to each immersion in either GO or the crosslinker. The dispersion of the GO nanosheets was enhanced by a 2-hour pre-fabrication sonication in a bath type sonicator (Ultrawave limited, $\mathrm{Hz}-(50-60)$ ). The films were fabricated under different dip-coating conditions i.e. 1, 5, 10 and 20 bi-layers and 1- and 5-minutes immersion times (see nomenclature in Table 1). Based on previous experience [15,22], other dip-assisted layer by layer operating conditions like the immersion and withdrawal speeds were kept constant at $2400 \mathrm{~mm} / \mathrm{min}$. 


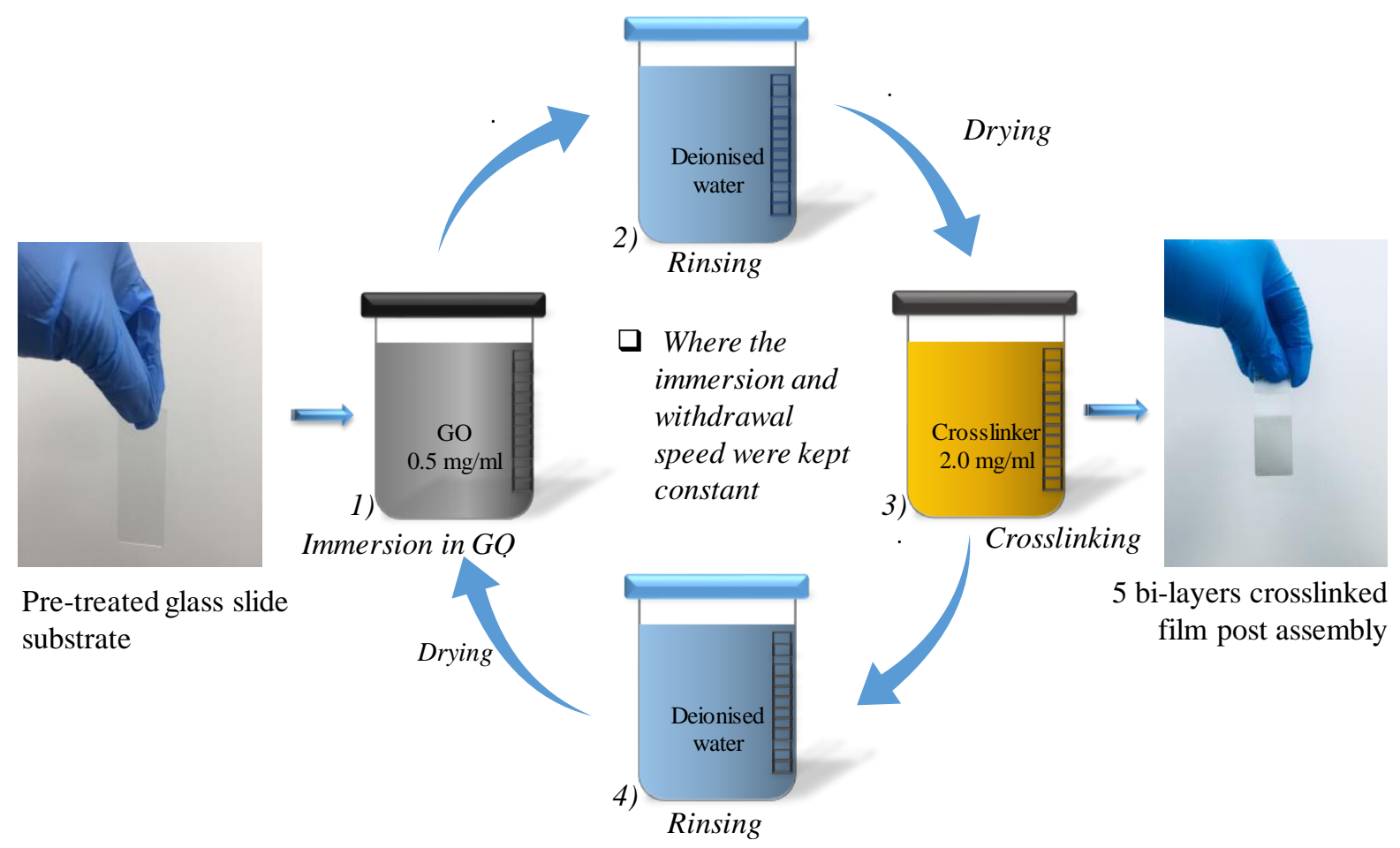

Figure 1. Dip-assisted layer by layer fabrication technique schematic.

Table 1. Nomenclature of the fabricated films at different immersion times and bi-layers.

\begin{tabular}{ccccc}
\hline & \multicolumn{5}{c}{ Number of bi-layers } \\
\hline $\begin{array}{c}\text { Immersion time } \\
(\text { min) }\end{array}$ & $\mathbf{1}$ & $\mathbf{5}$ & $\mathbf{1 0}$ & $\mathbf{2 0}$ \\
$\mathbf{1}$ & & & & \\
\cline { 2 - 5 } & GO-X-1'1 & GO-X-1'5 & GO-X-1'10 & GO-X-1'20 \\
$\mathbf{5}$ & GO-X-5'1 & GO-X-5'5 & GO-X-5'10 & GO-X-5'20
\end{tabular}

Key: $X=P E I$ or $P P D$.

\subsection{Post film fabrication characterizations}

The fabricated film surface coverage and homogeneity were characterised by means of a scanning electron microscope (SEM). The water contact angle measurements were subsequently recorded with a DMK 31BF03 camera and the angles were processed by image $\mathrm{j}$ software (1.50i/ Java 1.6.0) (Public Domain, BSD-2). To enhance the reliability of the results, an average of 10 contact angle measurements was taken. Pure water drops were introduced to the films in different positions from two different fabrication sets of the same film type. The thickness of the films on the other hand was evaluated by a Bruker DektakXT Profiling System 
(Stylus Profiler), Bruker's Vision64 software was then used to analyse the film thickness. Similarly, the reliability of the thickness results was enhanced by taking an average of 10 thickness measurements and noting the standard deviations of each recorded thickness in error bars.

\section{Results and discussion}

\subsection{Nature of interaction between GO and the crosslinkers}

Primarily, the nature of interaction between GO and the crosslinkers was determined and confirmed. This was significant as it is a precursor to intra(horizontal) and inter(vertical) sheet crosslinking during film fabrication [30].

The FTIR characterizations confirm the presence of oxygen containing functionalities in GO. For instance, the $\mathrm{O}-\mathrm{H}$ presence was verified by the absorption band at around $3340 \mathrm{~cm}^{-1}$, while the band at $1726 \mathrm{~cm}^{-1}$ is attributed to the $\mathrm{C}=\mathrm{O}$ stretching of ketone, carboxylic and/or ester groups (Figure 2). Furthermore, the peak at $1616 \mathrm{~cm}^{-1}$ corresponds to $\mathrm{C}=\mathrm{C}$ stretching vibrations and the band at $1060 \mathrm{~cm}^{-1}$ can be assigned to C-O (epoxy) groups [44-48].

The most significant functional groups present in the PPD spectrum are related to amine groups. Three bands between $3400-3000 \mathrm{~cm}^{-1}$ indicate the presence of primary amines [4547]. Additionally, the spectral bands at $1630 \mathrm{~cm}^{-1}$ and $1516 \mathrm{~cm}^{-1}$ have been correlated with $\mathrm{N}-\mathrm{H}$ deforming vibrations [52,53]. Finally, the band at $1250 \mathrm{~cm}^{-1}$ identifies the occurrence of $-\mathrm{C}-\mathrm{N}$ bonds in aromatic amines $[49,54]$ and the $-\mathrm{N}-\mathrm{H}$ wagging vibrations are distinguished by the sharp peak at $832 \mathrm{~cm}^{-1}[51]$.

The GO-PPD reacted entities illustrate that the characteristic triplet of primary amines in the $3400-3000 \mathrm{~cm}^{-1}$ region disappears at 1 and 5 minutes reaction times (Figure 2) and there is a small but very clear peak at $1510 \mathrm{~cm}^{-1}$ assigned to $-\mathrm{N}-\mathrm{H}$ bend [51,53]. These two facts suggest the existence of secondary amines [51], which consequently points to an epoxy ring opening reaction between GO and PPD at both reaction times [36] (Figure 3). 


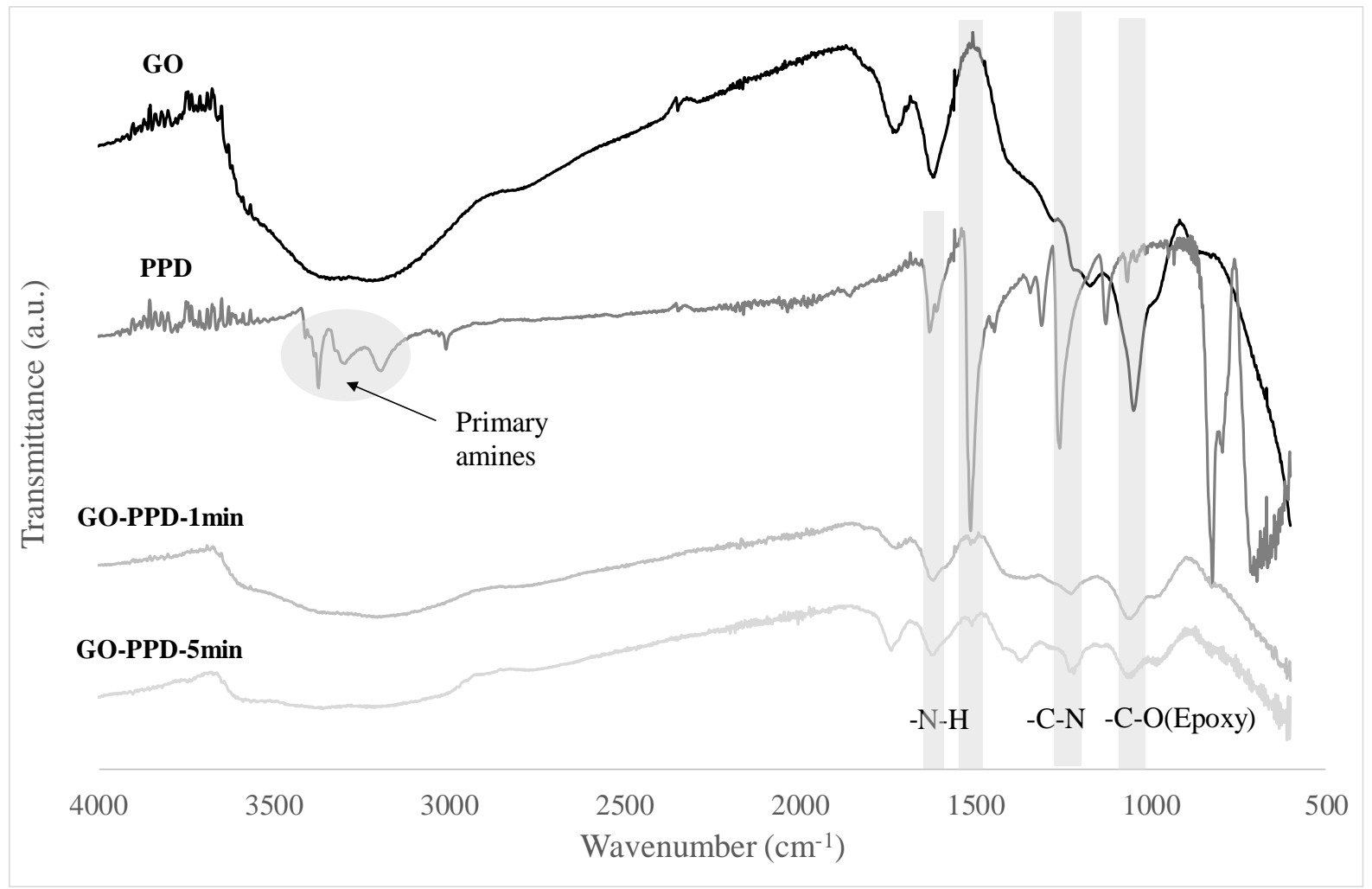

Figure 2. FTIR characterization spectra for the GO-PPD interactions after 1 and 5 minutes of reaction and the GO and PPD spectra.

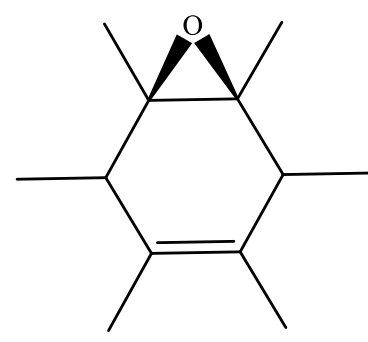

Epoxide group in GO<smiles>Nc1ccc(N)cc1</smiles>

PPD

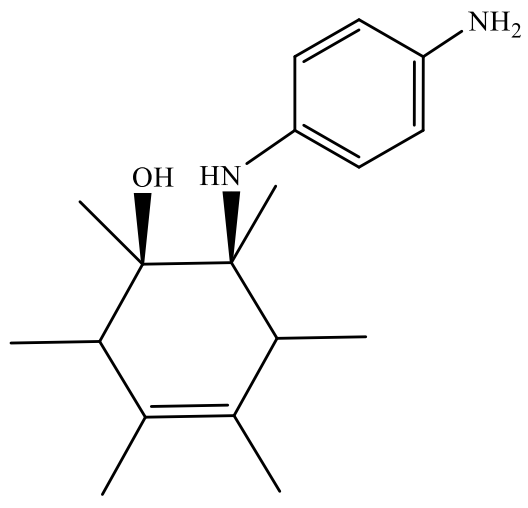

Interaction

Figure 3. The epoxy ring opening reaction between PPD and GO

The indication of the epoxy ring opening is further supported by the attenuation of the epoxy band at $1060 \mathrm{~cm}^{-1}$ (Figure 2), which is in agreement with the XPS results shown in Table 2, where a reduction in the epoxide proportion of the GO-PPD reacted entities (37\% GO vs $26 \%$ GO-PPD) is evident. Additionally, the reacted entities show the formation of a $-\mathrm{C}-\mathrm{N}$ band at 
$\sim 1220 \mathrm{~cm}^{-1}$ [49,54], which is also reflected in the XPS results, where the $\mathrm{C}=\mathrm{O} / \mathrm{C}-\mathrm{N}$ content increases from $1.2 \%$ up to $15.7 \%$ at 5 minutes reaction time.

Given the presence of different functional groups it is also most likely that other minor interactions like van der Waals forces and hydrogen bonding are occurring [29]. Structurally, $\mathrm{GO}$, in some areas where the oxygenated functional groups are not present, contains aromatic lattices and since PPD contains a benzene ring another possible interaction between GO and PPD is via the $\pi-\pi$ interactions [55,56].

Table 2. Surface chemistry of GO and GO-crosslinker reacted entities.

\begin{tabular}{lccccc}
\hline & GO & $\begin{array}{c}\text { GO-PPD } \\
\text { reacted 1 } \\
\text { min }\end{array}$ & $\begin{array}{c}\text { GO-PPD } \\
\text { reacted 5 } \\
\text { min }\end{array}$ & $\begin{array}{r}\text { GO-PEI } \\
\text { reacted 1 } \\
\text { min }\end{array}$ & $\begin{array}{c}\text { GO-PEI } \\
\text { reacted 5 } \\
\text { min }\end{array}$ \\
\hline $\mathrm{C} 1 \mathrm{~s}($ at.\%) & 71.2 & 70.1 & 67.5 & 67.5 & 61.9 \\
O1s (at.\%) & 27.4 & 27.6 & 29.9 & 23.0 & 26.6 \\
$\mathrm{~S} 1 \mathrm{~s}($ at.\%) & 1.4 & 1.6 & 1.6 & 2.6 & 3.0 \\
$\mathrm{~N} 1 \mathrm{~s}($ at.\%) & --- & 0.7 & 1.0 & 6.9 & 8.5 \\
$\mathrm{Csp}+\mathrm{Csp}(\%)$ & 58.5 & 57.8 & 51.9 & 58.2 & 52.3 \\
$\mathrm{C}(\mathrm{epoxy} / \mathrm{C}-\mathrm{OH} / \mathrm{C}=\mathrm{N}(\%)$ & 37.1 & 26.9 & 25.7 & 35.7 & 35.4 \\
$\mathrm{C}=\mathrm{O} / \mathrm{C}-\mathrm{N}(\%)$ & 1.2 & 9.3 & 15.7 & 2.0 & 8.0 \\
$\mathrm{COOH}(\%)$ & 3.2 & 5.0 & 6.3 & 4.1 & 4.1 \\
$\pi-\pi^{*}(\%)$ & 0.0 & 1.0 & 0.4 & 0.0 & 0.2 \\
\hline
\end{tabular}

On the other hand, PEI (Figure 4) contains a large amount of primary and secondary amine groups as well, which are evident in the region between $3400 \mathrm{~cm}^{-1}$ and $2900 \mathrm{~cm}^{-1}$ and by the strong $-\mathrm{N}-\mathrm{H}$ band at around $1640 \mathrm{~cm}^{-1}$ (Figure 5) [47, 48, 53]. The band at $1460 \mathrm{~cm}^{-1}$ corresponds to the $-\mathrm{CH}_{2}$ bending [51] and it can be also detected in the reacted entities (Figure $5)$.

In the GO-PEI reacted samples there is no disappearance of the band at $1060 \mathrm{~cm}^{-1}$, hence no indication of the epoxy ring opening reaction (see Figure 5). This is in accordance with the XPS results (Table 2) that show a constant percentage of epoxy groups regardless the reaction time (37.1\% in the GO vs $35.7 \%-35.4 \%$ in the reacted entities). Moreover, $\mathrm{pH}$ of GO suspension and PEI solution were 5.2 and 8.3 respectively, at these $\mathrm{pH}$ values both compounds have significant ionization and opposite charges [43,57], which supports the claim that their interaction is predominantly an electrostatic based one. 
<smiles>NCCNCCN(CCNCCN)CCN(CCNCCN)CCN(CCN)CCN</smiles>

Figure 4. PEI monomer (branched ethyleneimine)

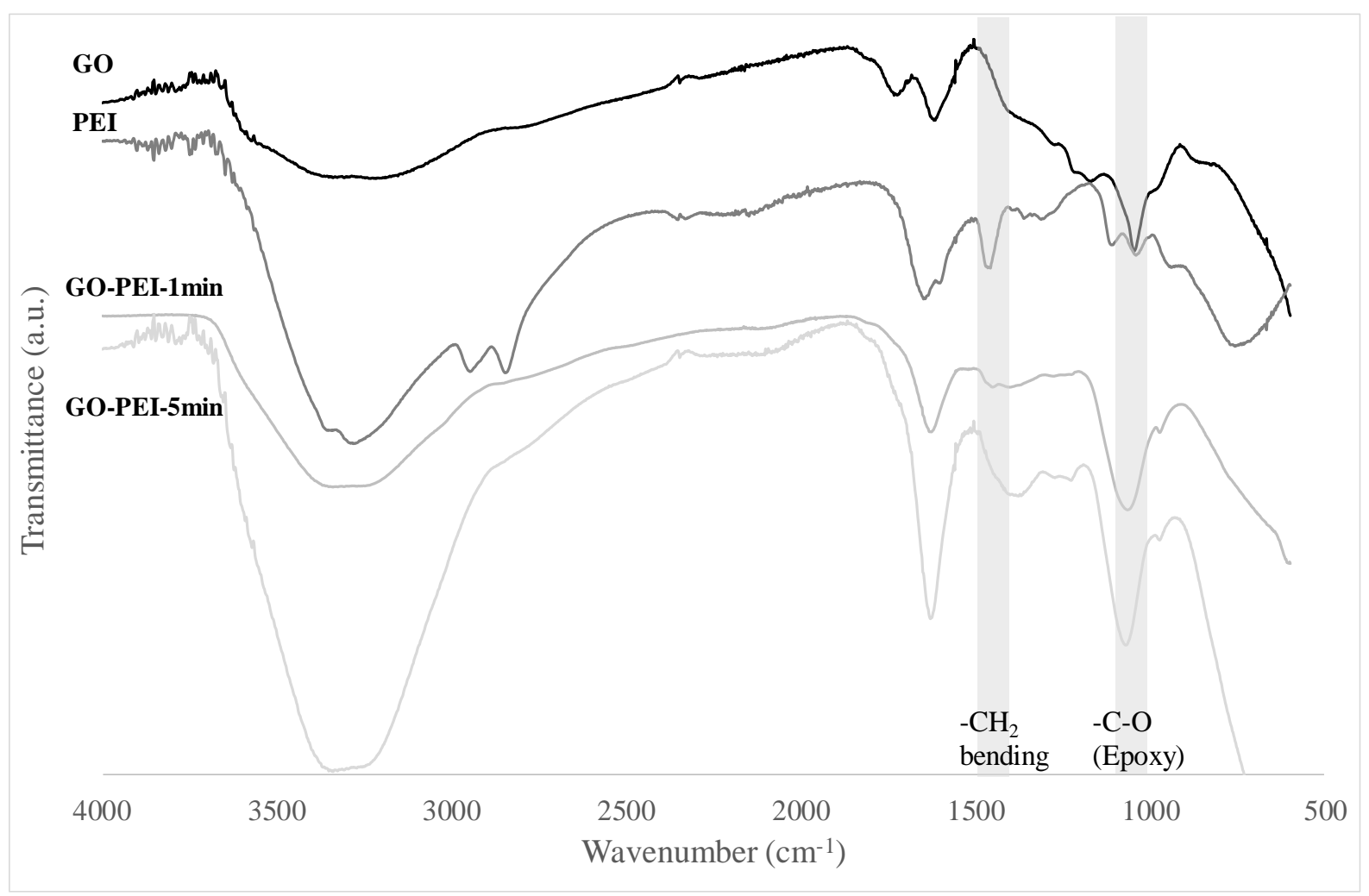

Figure 5. FTIR characterization spectra for the GO-PEI interactions after 1 and 5 minutes of reaction and the GO and PEI spectra

\subsection{Film continuity analysis}

Very good uniformity and homogeneity across the thin films can be observed in the pictures shown in Figure 6. The darkening of the glass slides indicates that coating and material assembly increase with the number of bi-layers and the immersion time in both sets. Uniform 
thickness of the coatings is to an extent confirmed by the homogeneous change in surface colour after all the different number of coating cycles.

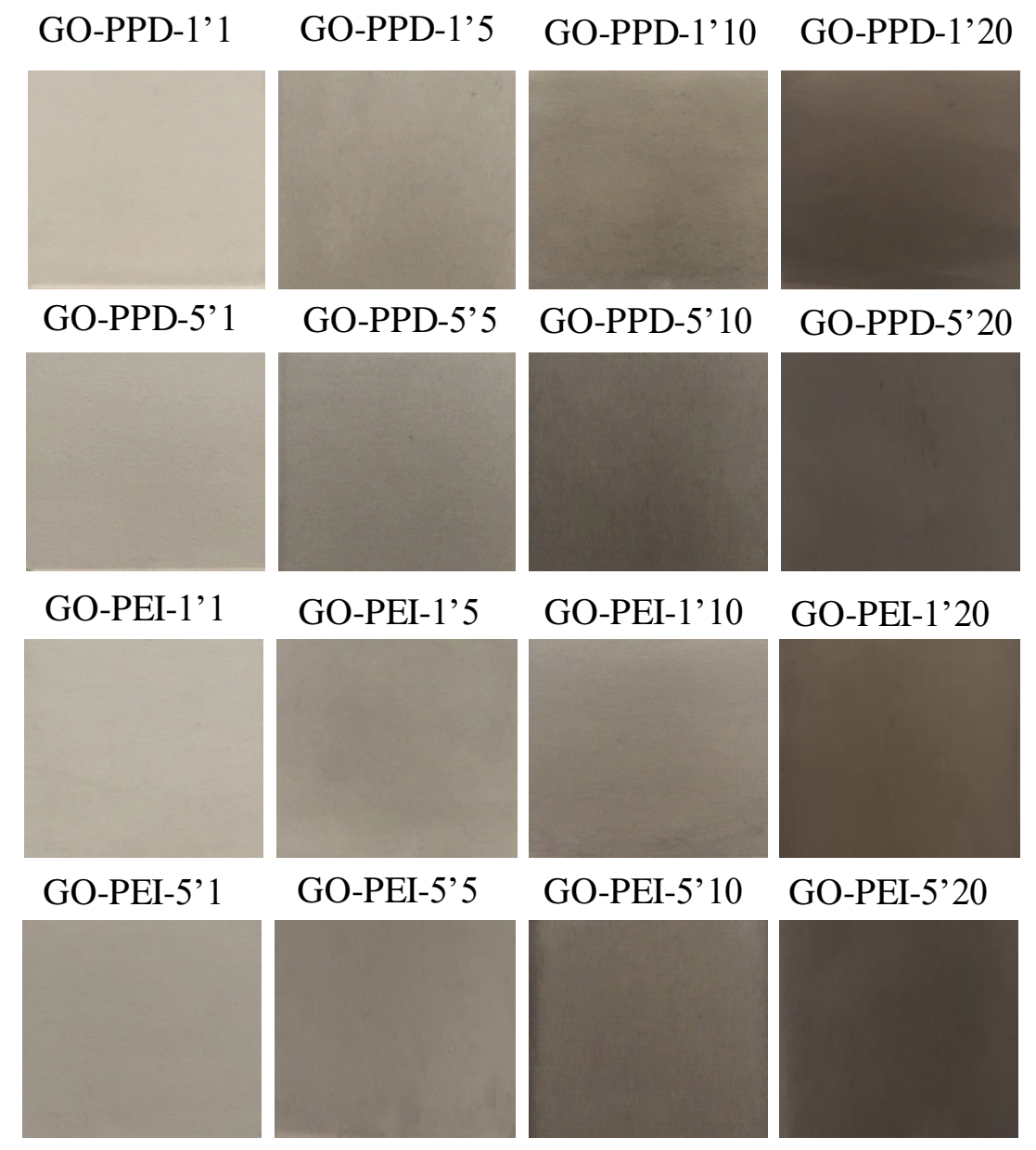

Figure 6. Camera pictures of the crosslinked thin films.

As it can be seen in further detail in Figure 7, excellent continuity coverage is achieved from the very first layer. Some wrinkles can be observed on the surface due to the folding of the GO nanosheets (Figure 7). 


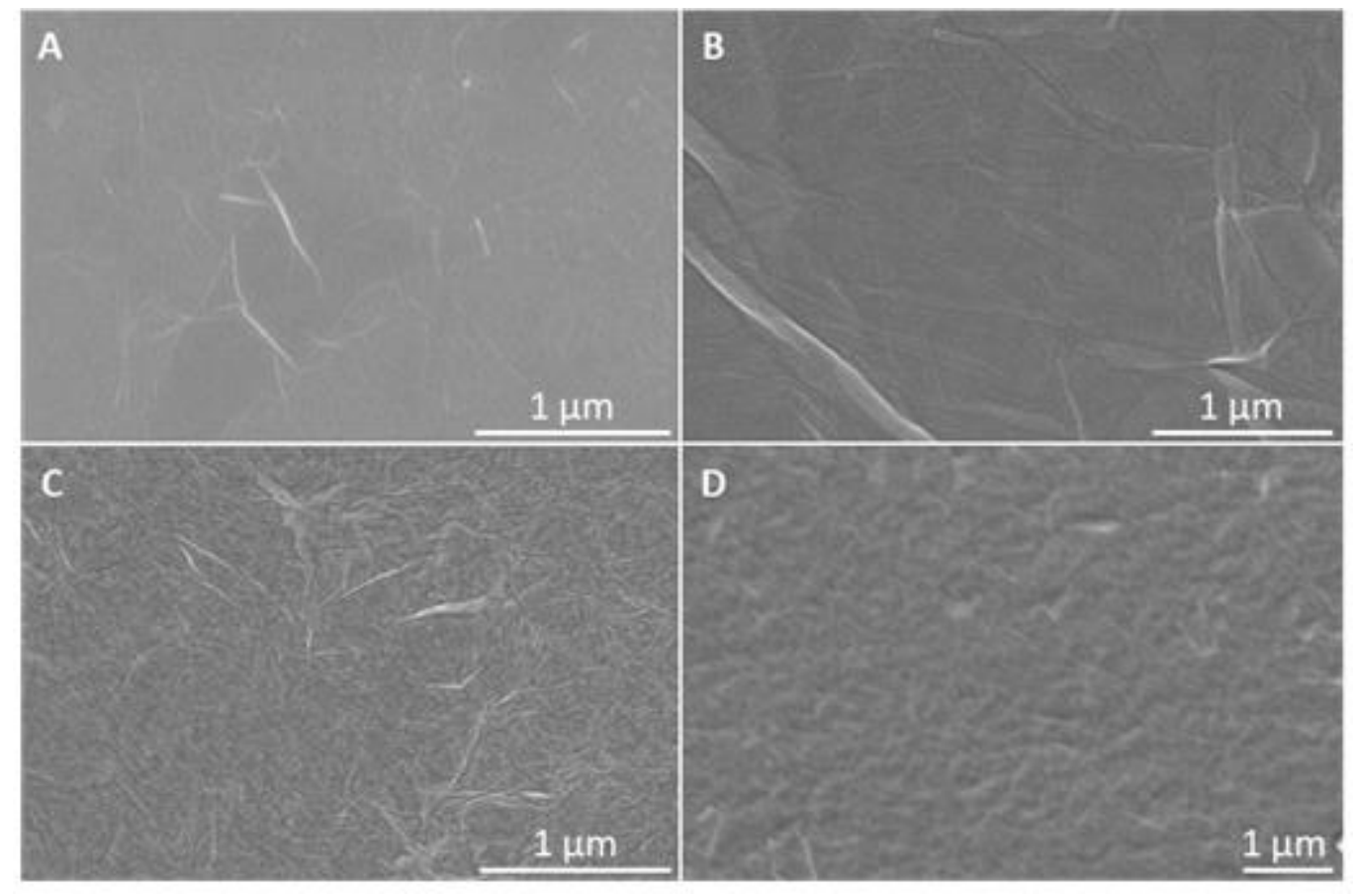

Figure 7. SEM images of the crosslinked thin films. A) GO-PPD 1'1; B) GO-PPD 1'20; C) GO-PEI 1'1; D) GO-PEI 1'20

\subsection{Thin film hydrophilicity}

Hydrophilicity is among the significant thin film characteristics. High film hydrophilicity can be of sentimental value or a limitation depending on the intended application of the fabricated films. For instance in surface coating lower film hydrophilicity is required while in water purification and desalination higher hydrophilicity is preferred [43,58]. Film hydrophilicity was evaluated by measurements of the water contact angles of the fabricated films (Figure 8). As aforementioned, 10 measurements in different positions from two differently fabricated films under the same operating conditions were undertaken and the average contact angle and standard deviations were noted. 


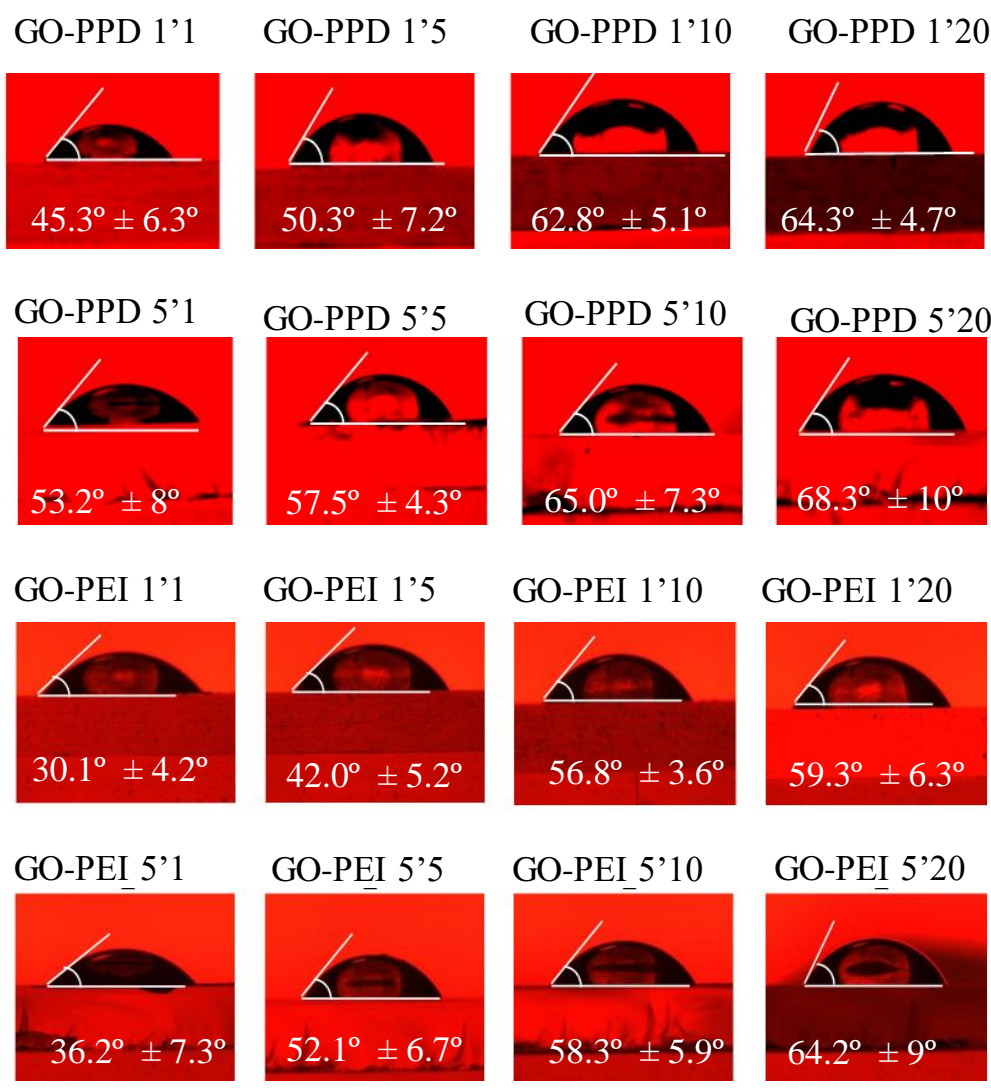

Figure 8. Water contact angles for the crosslinked films.

The general hydrophilicity trend shows that the water contact angle increases as the number of bi-layers increases. A notable decrease in hydrophilicity is observed from the first bi-layer to the 10th for both crosslinkers and eventually a gradual decrease from the 10th bi-layer to the 20th is observed at both immersion times for both crosslinkers (Figure 8).

The more hydrophobic nature of the crosslinkers in comparison to GO is the likely cause for the observed decrease in hydrophilicity of the fabricated films as the number of bi-layers and immersion time increases, owing to increased crosslinker accumulation fabrication.

Larger contact angles were recorded for the PPD crosslinked films in comparison to the PEI crosslinked films, which is due to the hydrophobic aromatic ring structure of the PPD monomer [59]. This is in agreement with the recorded magnitudes for the individual compounds $\left(68.2^{\circ} \pm 6.8^{\circ}\right.$ for PPD; $57.3^{\circ} \pm 4.3^{\circ}$ for pure PEI) and therefore proving the influence of the characteristics of the crosslinkers onto the overall hydrophilicity of the fabricated films. At 5 min deposition time and 20 bi-layers the contact angles reach the values of the pure crosslinkers or even exceed it in the case of the PEI $\left(64.2^{\circ} \pm 9^{\circ}\right.$ vs $\left.57.3^{\circ} \pm 4.3^{\circ}\right)$. This fact could be attributed to the reducing properties of PEI [60]. 


\subsection{Film thickness analysis}

The essentiality of ease of control of the thickness of the active layers cannot be understated in optimising device performances.

The thickness characterizations results show a strong linear correlation between film thickness and the respective number of bilayers at each of the immersion times for both sets of crosslinked films (Figure 9).

It is important to highlight that due to the thin thickness of the 1 bilayer films, especially those deposited at $1 \mathrm{~min}$ immersion time, it was necessary to fabricate them on a silicon wafer, as the roughness of the glass slides was overly and therefore it was not possible to have an accurate thickness measure for those specific samples.

Displayed in Figure 9 are the thickness results of the two sets of crosslinked films at both immersion times and different bilayer cycles. The results are very repeatable and 1 bilayer thin films at $1 \mathrm{~min}$ immersion are very close to the resolution of the equipment. 


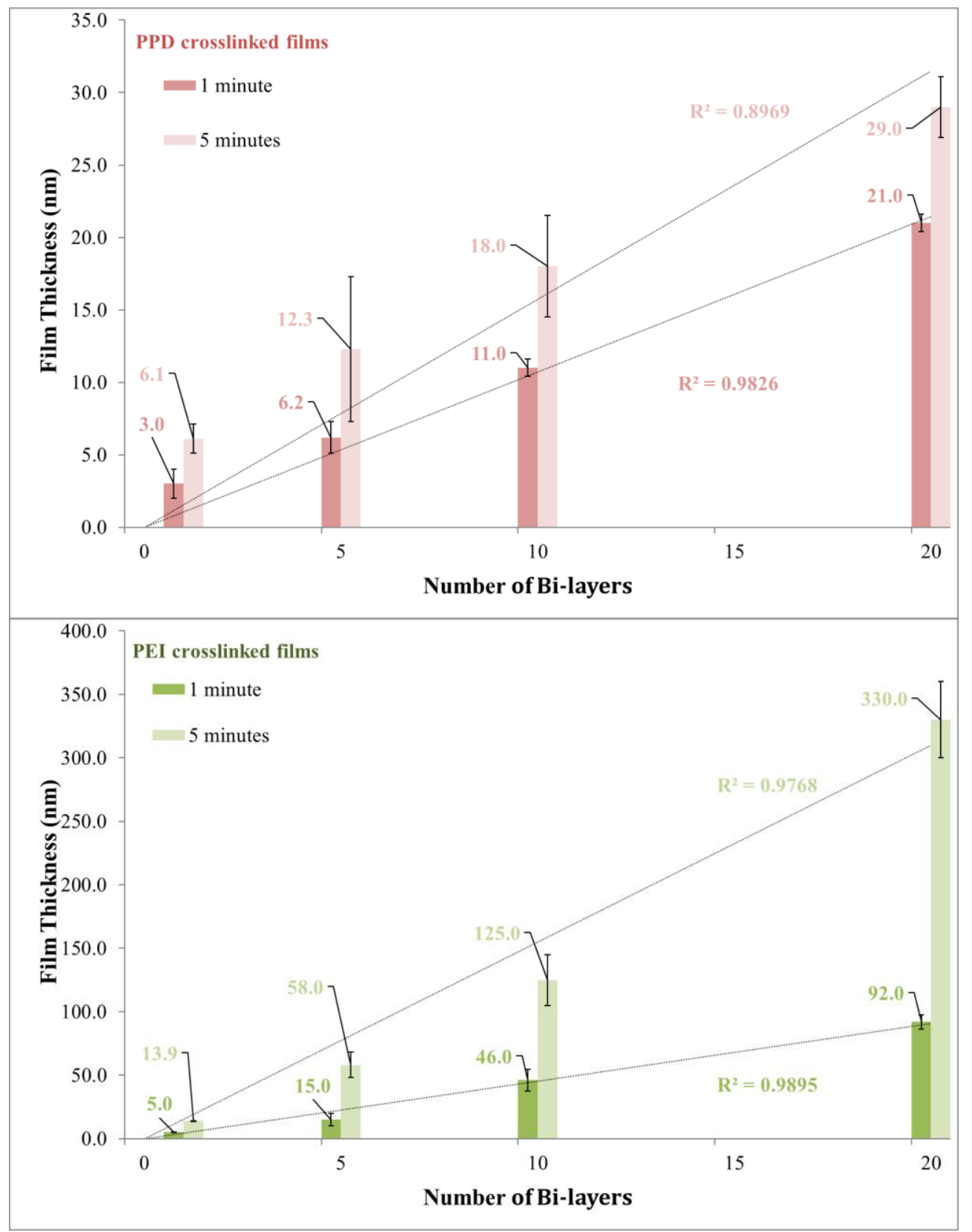

Figure 9. Thickness measurements of the cross-linked thin films.

A general linear trend due to the additional material accumulation as the immersion time and the number of bilayers increases is evident. Nevertheless, for the PEI-crosslinked films the thickness magnitude is much higher than that of the PPD thin films (one order of magnitude) as it can be seen in Figure 9. For instance, the PPD crosslinked films at 1 minute immersion time the thickness rises from $3 \mathrm{~nm}$ to $21 \mathrm{~nm}$ as the number of bi-layers increases from 1 to 20 bilayers, while it increases from $6 \mathrm{~nm}$ to $29 \mathrm{~nm}$ when the immersion time is increased to $5 \mathrm{~min}$. 
These values are relatively close to the theoretical expected step increase of $1.5 \mathrm{~nm}$ per bi-layer [58].

On the other hand, PEI crosslinked films thickness increases is from $5 \mathrm{~nm}$ to $92 \mathrm{~nm}$ (1 min dipping) and from $13.9 \mathrm{~nm}$ to $330 \mathrm{~nm}$ (5 min immersion time). Theoretically, since PEI is a polymer and therefore with varying chain lengths, the expected step increase for the PEI crosslinked films cannot be predicted with absolute certainty [61]. In both cases however a strong linear progression with the increasing number of bi-layers is observed.

The varying magnitude of increase in film thickness regarding the two crosslinkers is mainly due to the differences in the sizes of the crosslinkers, as PPD is a much smaller molecule while PEI is a bigger polymer with long branched chains and thus resulting in higher thickness as the number of bi-layers increases [61]. This further supports the claim that the thickness of the crosslinked films is majorly due to vertical inter layer crosslinking as evidenced by the fact that the bigger the crosslinker the higher the increase in film thickness magnitude.

The immersion time also has a significant impact on the thickness of the obtained films. In the case of PEI, increasing the immersion time from 1 minute to 5 minutes results in films three times thicker and between 1.5 and 2 times for PPD-crosslinked films. As such, selection of the interlayer crosslinker and the deposition parameters is key to fine-tuning and modification of the thickness of dip-assisted layer by layer assembled thin films.

The importance of the study is on the fact that the GO films can be easily fine-tuned to specific characteristics required in particular industrial operations. This makes the fabrication process economically efficient and viable. Furthermore, the successful fabrication of the different crosslinked thin films on glass slides highlights that various substrates including porous ones can be employed and as such the films can have different applications ranging from sensors to separation membranes and not solely in lab scale research applications. The study verifies the significance of both inter and intra layer crosslinking in altering the significant properties of GO based thin films. This further opens a possibility for the use of other different sized crosslinkers and polymers in fine tuning significant thin film characteristics via the dip-assisted layer-by layer-assembly.

\subsection{Conclusions}

In summation it was successfully demonstrated that crosslinked GO films with step-controlled thickness and hydrophilicity could be fabricated in a facile manner by means of dip-assisted 
layer-by-layer assembly. Good film homogeneity was also achieved. The thickness was successfully controlled through the use of different sized crosslinkers and operating conditions of the dip-assisted layer-by-layer method; the immersion time and the number of assembly cycles. It was observed that different crosslinkers have different impact on thickness progression as both the number of bi-layers and immersion time increase. This is advocated to the differences in size and nature of the crosslinkers entailed. Interpolation to fabrication operating conditions to obtain a specific characteristic can be thus be easily carried out.

\subsection{Acknowledgements}

The authors would like to extend their gratitude to the Botswana Government's Ministry of Tertiary Education and Scientific Research and the Top Achievers Scholarship Programme for offering a financial scholarship (100159844RA2 - TR. 163096) that aided the carrying out of this work. Authors also thank HarwellXPS EPSRC National Facility for the XPS characterizations.

\subsection{References}

[1] H. Frey, H.. Khan, Handbook of thin film technology: Applications and Developments of thin film technology, Springer Publishing, Berlin, Heidelberg, 2015.

[2] D.Ö. Thorsteinsson, J.T. Gudmundsson, Growth of HfN thin films by reactive high power impulse magnetron sputtering, AIP Adv. 8 (2018) 035124. doi:10.1063/1.5025553.

[3] G. Korotchenkov, Thin metal films". Handbook of Gas Sensor Materials. Integrated Analytical Systems, Springer Publishing, 2013.

[4] Y. Li, G. Xu, C. Cui, Y. Li, Flexible and Semitransparent Organic Solar Cells, Adv. Energy Mater. 8 (2018) 1-28. doi:10.1002/aenm.201701791.

[5] M.C. Rao, M.S. Shekhawat, a Brief Survey on Basic Properties of Thin Films for Device Application, Int. J. Mod. Phys. Conf. Ser. 22 (2013) 576-582. doi:10.1142/S2010194513010696.

[6] J. Liu, C. Wöll, Surface-supported metal-organic framework thin films: Fabrication methods, applications, and challenges, Chem. Soc. Rev. 46 (2017) 5730-5770. 
doi: $10.1039 / \mathrm{c} 7 \mathrm{cs} 00315 \mathrm{c}$.

[7] A.C. Ferrari, F. Bonaccorso, V. Fal'ko, K.S. Novoselov, S. Roche, P. Bøggild, S. Borini, F.H.L. Koppens, V. Palermo, N. Pugno, J.A. Garrido, R. Sordan, A. Bianco, L. Ballerini, M. Prato, E. Lidorikis, J. Kivioja, C. Marinelli, T. Ryhänen, A. Morpurgo, J.N. Coleman, V. Nicolosi, L. Colombo, A. Fert, M. Garcia-Hernandez, A. Bachtold, G.F. Schneider, F. Guinea, C. Dekker, M. Barbone, Z. Sun, C. Galiotis, A.N. Grigorenko, G. Konstantatos, A. Kis, M. Katsnelson, L. Vandersypen, A. Loiseau, V. Morandi, D. Neumaier, E. Treossi, V. Pellegrini, M. Polini, A. Tredicucci, G.M. Williams, B. Hee Hong, J.H. Ahn, J. Min Kim, H. Zirath, B.J. Van Wees, H. Van Der Zant, L. Occhipinti, A. Di Matteo, I.A. Kinloch, T. Seyller, E. Quesnel, X. Feng, K. Teo, N. Rupesinghe, P. Hakonen, S.R.T. Neil, Q. Tannock, T. Löfwander, J. Kinaret, Science and technology roadmap for graphene, related two-dimensional crystals, and hybrid systems, Nanoscale. 7 (2015) 4598-4810. doi:10.1039/c4nr01600a.

[8] V.B. Mohan, H. Souri, K. Jayaraman, D. Bhattacharyya, Mechanical properties of thin films of graphene materials: A study on their structural quality and functionalities, Curr. Appl. Phys. 18 (2018) 879-885. doi:10.1016/j.cap.2018.04.011.

[9] I. Levchenko, K.K. Ostrikov, J. Zheng, X. Li, M. Keidar, K.B.K. Teo, Scalable graphene production: Perspectives and challenges of plasma applications, Nanoscale. 8 (2016) 10511-10527. doi:10.1039/c5nr06537b.

[10] I.A. Vacchi, C. Spinato, J. Raya, A. Bianco, C. Ménard-Moyon, Chemical reactivity of graphene oxide towards amines elucidated by solid-state NMR, Nanoscale. 8 (2016) 13714-13721. doi:10.1039/c6nr03846h.

[11] Q. Tang, Z. Zhou, Z. Chen, Graphene-related nanomaterials: Tuning properties by functionalization, Nanoscale. 5 (2013) 4541-4583. doi:10.1039/c3nr33218g.

[12] L. Fernández-García, A.M. Pérez-Mas, P. Álvarez, C. Blanco, R. Santamaria, R. Menéndez, M. Granda, Morphological changes in graphene materials caused by solvents, Colloids Surfaces A Physicochem. Eng. Asp. 558 (2018) 73-79. doi:10.1016/j.colsurfa.2018.08.030.

[13] Z. González, A.M. Pérez-Mas, C. Blanco, M. Granda, R. Santamaría, Influence of the electrophoretic deposition parameters on the formation of suspended graphene-based films., Mater. Des. 160 (2018) 58-64. https://doi.org/10.1016/j.matdes.2018.08.063. 
[14] M. Hernaez, A.G. Mayes, S. Melendi-Espina, Graphene Oxide in Lossy Mode Resonance-Based Optical Fiber Sensors for Ethanol Detection, Sensors. 18 (2018). doi:10.3390/s18010058.

[15] V. Kandjou, M. Hernaez, B.M. Acevedo, A.M. Perez-Mas, S. Melendi-Espina, The fabrication of controlled thickness graphene oxide films by means of dip-assisted layer by layer assembly, in: World Conf. Carbon, Madrid, 2018: p. Paper 0649.

[16] D.R. Dreyer, S. Park, C.W. Bielawski, R.S. Ruoff, The chemistry of graphene oxide, R. Soc. Chem. 39 (2010) 228-240. doi:10.1039/b917103g.

[17] N.I. Zaaba, K.L. Foo, U. Hashim, S.J. Tan, W.W. Liu, C.H. Voon, Synthesis of Graphene Oxide using Modified Hummers Method: Solvent Influence, Procedia Eng. 184 (2017) 469-477. doi:10.1016/j.proeng.2017.04.118.

[18] H. Yu, B. Zhang, C. Bulin, R. Li, R. Xing, High-efficient Synthesis of Graphene Oxide Based on Improved Hummers Method, Sci. Rep. 6 (2016) 1-7. doi:10.1038/srep36143.

[19] M. Hernaez, B. Acevedo, A.G. Mayes, S. Melendi-Espina, High-performance optical fiber humidity sensor based on lossy mode resonance using a nanostructured polyethylenimine and graphene oxide coating, Sensors Actuators, B Chem. (2019) $408-414$.

[20] M. Hernaez, S. Melendi-Espina, A.G. Mayes, Lossy Mode Resonance Generation by Graphene Oxide Coatings onto Cladding-Removed Multimode Optical Fiber, IEEE Sensors. (2019). doi:10.1109/JSEN.2019.2906010.

[21] D.A. Dikin, S. Stankovich, E.J. Zimney, R.D. Piner, G.H.B. Dommett, G. Evmenenko, S.T. Nguyen, R.S. Ruoff, Preparation and characterization of graphene oxide paper, Nature. 448 (2007) 457-460. doi:10.1038/nature06016.

[22] V. Kandjou, A.M. Perez-mas, B. Acevedo, M. Hernaez, A.G. Mayes, S. Melendiespina, Enhanced covalent p-phenylenediamine crosslinked graphene oxide membranes : Towards superior contaminant removal from wastewaters and improved membrane reusability, J. Hazard. Mater. 380 (2019) 120840. doi:10.1016/j.jhazmat.2019.120840.

[23] M. Hernaez, S. Melendi-Espina, A.G. Mayes, Sensitivity enhancement of lossy mode resonance-based ethanol sensors by graphene oxide coatings, in: IEEE Sensors Conf., Glasgow, 2017: p. 1450. doi:10.1109/ICSENS.2017.8234320. 
[24] P. Sanchez, C. Hernaez, Miguel Zamarreño, Arregui, A. Mayes, S. Melendi-Espina, Optical fiber exhaled breath sensor based on lossy mode resonance using a graphene oxide sensitive coating, in: 5th Int. Symp. Sens. Sci. I3S 2017, 2017: p. 713. doi:10.3390/proceedings1080713.

[25] P. Sanchez, V. Kandjou, M. Hernaez, F.. Zamarreño, C.R Arregui, A.. Mayes, S. Melendi-Espina, Graphene oxide film fabrication with controlled thickness on siliconbased substrates, in: World Conf. Carbon, 2017: p. 302.

[26] S. Basu, P. Bhattacharyya, Recent developments on graphene and graphene oxide based solid state gas sensors, Sensors Actuators, B Chem. 173 (2012) 1-21. doi:10.1016/j.snb.2012.07.092.

[27] H. Li, Z. Song, X. Zhang, Y. Huang, S. Li, Y. Mao, Ultrathin, Molecualr-sieving graphene oxide membranes for selective hydrogen separation, Science (80-. ). 342 (2013) 95-98.

[28] A. Lerf, H. He, M. Forster, J. Klinowski, Structure of Graphite Oxide Revisited ", J. Phys. Chem. B. 102 (1998) 4477-4482. doi:10.1021/jp9731821.

[29] W. Gao, Graphene Oxide: Reduction Recipes, Spectroscopy, and Applications, Springer, Cham, 2015. doi:https://doi.org/10.1007/978-3-319-15500-5.

[30] Z. Jia, Y. Wang, Covalently crosslinked graphene oxide membranes by esterification reactions for ions separation, J. Mater. Chem. A. 3 (2015) 4405-4412. doi:10.1039/c4ta06193d.

[31] Z. Jia, Y. Wang, W. Shi, J. Wang, Diamines cross-linked graphene oxide free-standing membranes for ion dialy sis separation, J. Memb. Sci. 520 (2016) 139-144. doi:10.1016/j.memsci.2016.07.042.

[32] H. Shi, C. Wang, Z. Sun, Y. Zhou, K. Jin, G. Yang, Transparent conductive reduced graphene oxide thin films produced by spray coating, Sci. China Physics, Mech. Astron. 58 (2015) 1-5. doi:10.1007/s11433-014-5614-y.

[33] A.A. Green, M.C. Hersam, Solution phase production of graphene with controlled thickness via density differentiation, Nano Lett. 9 (2009) 4031-4036. doi:10.1021/n1902200b.

[34] J.J. Richardson, J. Cui, M. Björnmalm, J.A. Braunger, H. Ejima, F. Caruso, Innovation in Layer-by-Layer Assembly, Chem. Rev. 116 (2016) 14828-14867. 
doi:10.1021/acs.chemrev.6b00627.

[35] M. Hu, B. Mi, Layer-by-layer assembly of graphene oxide membranes via electrostatic interaction, J. Memb. Sci. 469 (2014) 80-87. doi:10.1016/j.memsci.2014.06.036.

[36] M.M. Sk, C.Y. Yue, Layer-by-layer (LBL) assembly of graphene with pphenylenediamine (PPD) spacer for high performance supercapacitor applications, RSC Adv. 4 (2014) 19908-19915. doi:10.1039/c4ra02652g.

[37] M.I. Verkhovsky, A. Jasaitis, M.L. Verkhovskaya, J.E. Morgan, M. Wikström, Proton translocation by cytochrome c oxidase., Nature. 400 (1999) 480-483. doi:10.1038/22813.

[38] M. Frasconi, R. Tel-vered, J. Elbaz, I. Willner, Electrochemically-Stimulated pH Changes : A Route to Control Chemical Reactivity, J. Am. Chem. Soc. 132 (2010) 13.

[39] A.P. Dementjev, A. de Graaf, M.C.. van de Sanden, A.. Maslakov, K.I Naumkin, A.. Serov, X-Ray photoelectron spectroscopy reference data for identification of the C3 N4 phase in carbon-nitrogen films., Diam. Relat. Mater. 9 (2000) 1904-1907.

[40] S. Bhattacharyya, C. Cardinaud, G. Turban, Spectroscopic determination of the structure of amorphous nitrogenated carbon films, J. Appl. Phys. 83 (1998) 4491.

[41] S. Bhattacharyya, J. Hong, G. Turban, Determination of the structure of amorphous nitrogenated carbon films by combined Raman and x-ray photoemission spectroscopy, J. Appl. Phys. 83 (1998) 3917.

[42] S.D. Chandradoss, A.C. Haagsma, Y.K. Lee, J.-H. Hwang, J.-M. Nam, C. Joo, Surface Passivation for Single-molecule Protein Studies, J. Vis. Exp. (2014) 1-8. doi:10.3791/50549.

[43] Q. Nan, P. Li, B. Cao, Fabrication of positively charged nanofiltration membrane via the layer-by-layer assembly of graphene oxide and polyethylenimine for desalination, Appl. Surf. Sci. 387 (2016) 521-528. doi:10.1016/j.apsusc.2016.06.150.

[44] M. Aleksandrzak, P. Adamski, W. Kukulka, B. Zielinska, E. Mijowska, Effect of graphene thickness on photocatalytic activity of $\mathrm{TiO} 2$ - graphene nanocomposites, Appl. Surf. Sci. 331 (2015) 193-199.

[45] G. Wang, B. Wang, J. Park, J. Yang, X. Shen, J. Yao, Synthesis of enhanced 
hydrophilic and hydrophobic graphene oxide nanosheets by a solvothermal method, Carbon N. Y. 7 (2008) 6-10. doi:10.1016/j.carbon.2008.09.002.

[46] C. Hontoria-Lucas, A.. Lopez-Peinado, de D. Lopez-Gonzalez, M.. Cervantes-Rojas, R.. Martin-Aranda, Study of oxygen containing groups in a series of graphite oxides: Physical and Chemical Characterization, Carbon N. Y. 95 (1995).

[47] D.C. Marcano, D. V Kosynkin, J.M. Berlin, A. Sinitskii, Z. Sun, A. Slesarev, L.B. Alemany, W. Lu, J.M. Tour, Improved Synthesis of Graphene Oxide, ACS Nano. 4 (2010). doi:10.1021/nn1006368.

[48] J.I. Paredes, S. Villar-Rodil, A. Martyinez-Alonso, J.M.D. Tascon, Graphene Oxide Dispersions in Organic Solvents Graphene, Langmuir. 24 (2008) 10560-10564. doi:10.1021/la801744a.

[49] X. Li, M. Huang, Y. Yang, Synthesis and characterization of o -phenylenediamine and xylidine copolymers, 42 (2001).

[50] R.H. Sestrem, D.C. Ferreira, R. Landers, M.L.A. Temperini, M. Gustavo, Structure of chemically prepared poly- ( para -phenylenediamine ) investigated by spectroscopic techniques, Polymer (Guildf). 50 (2009) 6043-6048. doi:10.1016/j.polymer.2009.10.028.

[51] N.P.G. Roeges, Guide to the complete interpretation of infrared spectra of organic structures, John Wiley and Sons Ltd. Baffins Lane, Chichester, West Sussex, 1994.

[52] R.A. Heacock, L. Marion, The infrared spectra of secondary amines and their salts, Can. J. Chem. 34 (2011) 1782-1795.

[53] A.P. Cleaves, E.K. Plyler, The Infra-Red Absorption Spectrum of Methylamine Vapor, J. Chem. Phys. 7 (1939) 563-569.

[54] R.M. Silverstein, F.X. Webster, D.J. Kiemle, Identification of organic compounds (Spectrometric Identification of Organic Compounds. 4th ed), 4th ed., Wiley, New York, 1981.

[55] J. Li, X. Zeng, T. Ren, E. van der Heide, The Preparation of Graphene Oxide and Its Derivatives and Their Application in Bio-Tribological Systems, Lubricants. 2 (2014) 137-161. doi:10.3390/lubricants2030137.

[56] S. Dai, Z. Xu, M. Zhu, Y. Qian, C. Wang, Detection of p-phenylenediamine based on a 
glassy carbon electrode modified with nitrogen doped graphene, Int. J. Electrochem. Sci. 10 (2015) 7063-7072.

[57] L. Zhao, H. Zhang, N. Hoon, D. Hui, J. Hee, Q. Li, H. Sun, P. Li, Preparation of graphene oxide / polyethyleneimine layer-by-layer assembled fi $\mathrm{lm}$ for enhanced hydrogen barrier property, 92 (2016) 252-258.

doi:10.1016/j.compositesb.2016.02.037.

[58] T. Lee, S.H. Min, M. Gu, Y.K. Jung, W. Lee, J.U. Lee, D.G. Seong, B.S. Kim, Layerby-Layer Assembly for Graphene-Based Multilayer Nanocomposites: Synthesis and Applications, Chem. Mater. 27 (2015) 3785-3796.

doi:10.1021/acs.chemmater.5b00491.

[59] W.S. Hung, C.H. Tsou, M. De Guzman, Q.F. An, Y.L. Liu, Y.M. Zhang, C.C. Hu, K.R. Lee, J.Y. Lai, Cross-linking with diamine monomers to prepare composite graphene oxide-framework membranes with varying d-spacing, Chem. Mater. 26 (2014) 2983-2990. doi:10.1021/cm5007873.

[60] H. Liu, K. Tapas, N.H. Kim, B.-C. Kud, J.H. Lee, In situ synthesis of the reduced graphene oxide-polyethyleneimine composite and its gas barrier properties, J. Mater. Chem. A. 1 (2013) 3739-3746. doi:10.1039/C3TA01228J.

[61] C.N. Lungu, M. V. Diudea, M. V. Putz, I.P. Grudziński, Linear and branched PEIs (Polyethylenimines) and their property space, Int. J. Mol. Sci. 17 (2016). doi:10.3390/ijms17040555. 